\title{
Elastic Model Based Non-rigid Registration Incorporating Statistical Shape Information
}

\author{
Yongmei Wang* and Lawrence H. Staib*+ \\ Departments of Electrical Engineering* and Diagnostic Radiology ${ }^{+}$ \\ Yale University, P.O. Box 208042, New Haven, CT 06520-8042 \\ wang@noodle.med.yale.edu, lawrence.staib@yale.edu
}

\begin{abstract}
This paper describes a new method of non-rigid registration using the combined power of elastic and statistical shape models. The transformations are constrained to be consistent with a physical model of elasticity to maintain smoothness and continuity. A Bayesian formulation, based on this model, on an intensity similarity measure, and on statistical shape information embedded in corresponding boundary points, is employed to find a more accurate and robust non-rigid registration. A dense set of forces arises from the intensity similarity measure to accommodate complex anatomical details. A sparse set of forces constrains consistency with statistical shape models derived from a training set. A number of experiments were performed on both synthetic and real medical images of the brain and heart to evaluate the approach. It is shown that statistical boundary shape information significantly augments and improves elastic model based non-rigid registration. ${ }^{1}$
\end{abstract}

\section{Introduction}

Comparing function or morphology between individuals requires non-rigid registration, because the detailed anatomical structure differs, sometimes greatly, between individuals. The goal of our non-rigid registration is to remove structural variation between individuals by matching an atlas image to each individual, or study, image, in order to have a common coordinate system for comparison. Shape differences between the atlas and study's anatomy are contained in the non-rigid transformation.

There have been many approaches to non-rigid registration in recent years [2] [3] [4] [6] [8] [9] [10] [15]. Usually, the transformation is constrained in some way because of the ill-posedness (i.e. in this case, the existence of many possible solutions) of the problem. Physical models, for example, linear elastic models, are widely used to enforce topological properties on the deformation and then constrain the enormous solution space [2] [4] [8] [9] [10]. Here, we are particularly interested in intensity based deformation using elastic models. Our goal is to incorporate statistical shape information into this type of elastic model based registration and to develop a more accurate and robust algorithm.

Christensen et al. [4] present two physical models for non-rigid registration of the brain. The transformations are constrained to be consistent with the physical properties of deformable elastic solids in the first method and those of viscous

\footnotetext{
${ }^{1}$ This work was supported in part by a grant from the Whitaker Foundation.
} 
fluids in the second. Viscous fluid models are less constraining than elastic models and allow long-distance, nonlinear deformations of small subregions. In these formulations, however, no matter what model is used, elastic solid [10], viscous fluid [5], or other physics model such as hyperelasticity [11], the deformed configuration of the atlas is always determined by driving the deformation using only pixel-by-pixel intensity difference between images. In many applications, however, this kind of warping is under-constrained and admits to unreasonable registration. Corresponding anatomical structure may shift or twist away from one position to another (Fig.1(a)(b)). Even if the driving force is very small, the transformation may not be accurate enough, or may even be completely wrong, even though the deformed atlas and study appear similar (Fig.1(b)(c)(d)). In these circumstances, if shape information had been included, the correct mapping or registration could have been found (Fig.1(g)(h)). In addition, due to the use of the gray-level gradient of the deformed atlas in the body force formulation [4], lower contrast objects deform much slower than high-contrast objects, independent of their importance. Sometimes objects do not deform well because their gradient is too low compared to high-gradient objects (Fig.2(c)(d)). With the incorporation of the shape information, the result is improved (Fig.2(h)).

(a)

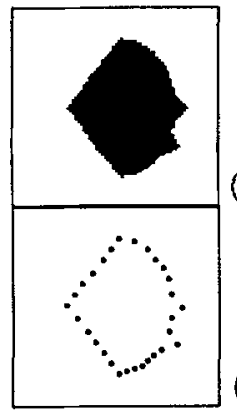

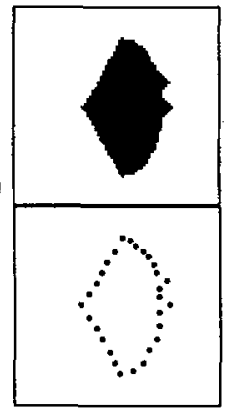

(c)

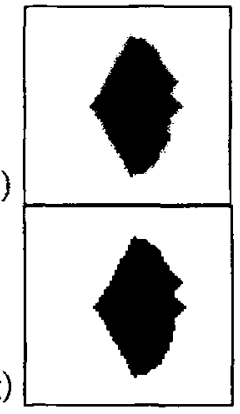

(d)

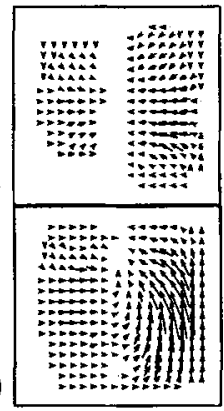

Fig. 1. Comparison of synthetic image $(64 \times 64)$ non-rigid registration methods. (a): atlas image; (b): study image; (c): deformed atlas image by Christensen\&Miller's elastic method [4] [10] (based on our implementation, discussed in Section 5); (d): corresponding unreasonable vector map of (c) (The two bumps on the right side do not slide up to the corresponding bumps, as desired); (e): atlas image control points; (f): study image control points; $(\mathrm{g})$ : deformed atlas image by our method; $(\mathrm{h})$ : vector map of our elastic transformation showing correct tracking of features.

Davatzikos and Prince [8] propose a method where they first identify the boundaries of homologous brain regions in two images to be registered (e.g., cortex, ventricles, etc.), and establish a one-to-one mapping between them. Based on this mapping, they deform the boundaries in one image into those in the other image. The rest of the image is deformed by solving the equations describing the deformation of an elastic body using the boundary deformation as input. In this approach, although the mapping may be accurate on the boundary, the farther away the structure is from the boundary, the more error there is, because only information from object boundaries is used for registration. Also, the localization 
(a)

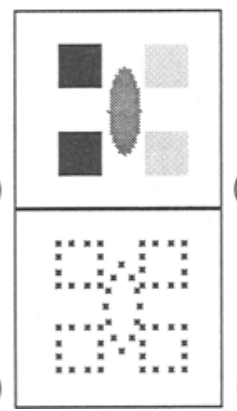

(b)

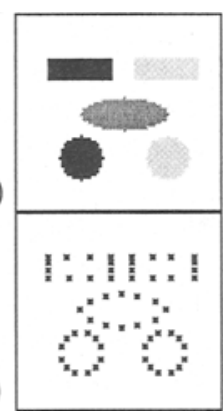

(c)

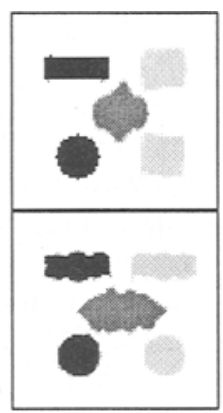

(d)

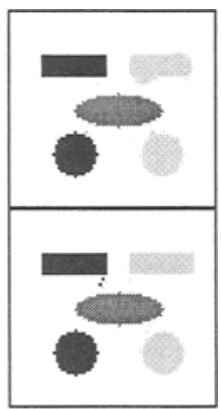

Fig. 2. Comparison of synthetic multi-gradient image $(64 \times 64)$ non-rigid registration methods. (a): atlas image; (b): study image; (c): deformed atlas at iteration 20 by Christensen\&Miller's elastic method [4] [10] ; (d): deformed atlas at iteration 150 by Christensen\&Miller's elastic method; (e): atlas image control points; (f): study image control points; $(\mathrm{g})$ : deformed atlas at iteration 150 by Davatzikos's elastic method [8], with body force solely determined by corresponding pairs of control points; (h): deformed atlas at iteration 150 by our elastic method showing good convergence without any boundary jiggling effect.

of the object boundary depends on the density of the boundary control points. The resulting boundaries can have quite severe errors producing a jiggling effect when the boundary control points are not dense enough, as shown in Fig.2(g). In our approach, we also use intensity information and thus the density of the control points is not as important (Fig.2(h)). In addition, the boundary information used in their approach is derived by an active contour algorithm [8], and it does not include any shape information which we believe is crucial in non-rigid registration for medical images.

The algorithm we employed is based on an elastic model, a gray level similarity measure and a consistency measure between corresponding boundary points. The statistical shape information is embedded in the boundary finding with correspondence process [16] applied to the study. This method uses statistical point models with shape, and shape variation, generated from sets of examples by principal component analysis of the covariance matrix. The power of elastic and statistical shape models are combined in our approach using a Bayesian framework.

\section{Elastic Model}

There is no true elastic model for deformation because, for example, one anatomical structure does not literally result from the deformation of another structure. We use an analogous linear elastic model to control the deformation. Without it, the results could be almost completely arbitrary.

An Eulerian reference is used in our elastic model formulation. In this frame, a particle is tracked with respect to its final coordinates. The non-rigid registration is defined by the transformation corresponding to a homeomorphic mapping of the coordinate system, defined in $2 \mathrm{D}$ by:

$$
\boldsymbol{w}=(x, y) \rightarrow\left(x-u_{x}(w), y-u_{y}(w)\right)
$$


where $u(\boldsymbol{w})=\left[u_{x}(\boldsymbol{w}), u_{y}(\boldsymbol{w})\right]^{T}$ is the displacement at each pixel $\boldsymbol{w}$ whose coordinate is denoted as $(x, y)$. This mapping allows for the detailed local transformation into the specific anatomy of the individual, or study.

We want to find this transformation that best matches the atlas with the study, constrained by the elastic model. The formulation of the elastic model is similar to that in [4]. For linear elastic solids, the force is proportional to the displacement. The spatial transformation satisfies the partial differential equation $(\mathrm{PDE})$ :

$$
\mu \nabla^{2} u+(\mu+\beta) \nabla(\nabla \cdot u)=\boldsymbol{F}(u)
$$

with certain boundary conditions such as that $u(w)=0$ for $\boldsymbol{w}$ on the image boundary. In this equation, $\mu$ and $\beta$ are Lamé constants. The body force, $\boldsymbol{F}(\boldsymbol{u})$, is the driving function that deforms the atlas into the shape of the study, which will be formulated in detail in Section 4 .

\section{Statistical Shape Model Based Boundary Finding}

Numerous deformable model based boundary finding methods have been proposed [7] [13] [14]. We have developed a statistical shape model based boundary finding with correspondence algorithm, which has been described in detail in [16]. Global shape parameters derived from the statistical variation of object boundary points in a training set are used to model the object [7]. A Bayesian formulation, based on this prior knowledge and the edge information of the input image, is employed to find the object boundary with its subset points in correspondence with the point sets of boundaries in the training set.

Given $m$ aligned examples, and for each example, a set of $N$ labeled points, $\mathbf{L}_{i}=\left(x_{i}(1), y_{i}(1), x_{i}(2), y_{i}(2), \cdots, x_{i}(N), y_{i}(N)\right)^{T},(i=1,2, \cdots, m)$, we calculate the mean shape, $\mathbf{L}$, and the covariance about the mean, $C_{\text {training }}$. The $t$ eigenvectors $\mathbf{q}_{i}$ of $C_{\text {training }}$ corresponding to the largest $t$ eigenvalues, $\lambda_{i}$, give a set of uncorrelated basis vectors, or modes of variation, for a deformable model. A new example can be calculated using $\mathbf{L}=\overline{\mathbf{L}}+Q \mathbf{a}$, where $Q=\left(\mathbf{q}_{1}\left|\mathbf{q}_{2}\right| \cdots \mid \mathbf{q}_{t}\right)$ is the matrix of eigenvectors and $\mathbf{a}=\left(a_{1}, a_{2}, \cdots, a_{t}\right)^{T}$ is the vector of weights, or shape parameters,to be determined. As a varies from zero, the corresponding shapes will be similar to those in the training set.

Given the statistical models, our aim is to match them to particular examples of structure in the individual images, and find the shape parameters, $\mathbf{a}=\left(a_{1}, a_{2}, \cdots, a_{t}\right)^{T}$, and pose parameters: scale $s$, rotation $\theta$, and translation $T_{x}, T_{y}$. The combined pose and shape parameter vector to be determined is $\mathbf{p}=\left(s, \theta, T_{x}, T_{y}, a_{1}, a_{2}, \ldots, a_{t}\right)$.

A Bayesian formulation leads to $([16])$ :

$$
M(\mathbf{p})=\sum_{j=1}^{t+4}\left[-\frac{\left(p_{j}-m_{j}\right)^{2}}{2 \sigma_{j}^{2}}\right]+\frac{1}{\sigma_{n}^{2}} \sum_{n=1}^{N} E(x(\mathbf{p}, n), y(\mathbf{p}, n))
$$

where $m_{j}$ is the mean of $p_{j} ; \sigma_{j}$ is the standard deviation for each of the parameters and $\sigma_{n}$ is the standard deviation of the white zero mean Gaussian noise associated with the image noise model [16]. This equation is the maximum $a$ posteriori objective incorporating a prior bias to likely shapes and poses (first 
term) and a match to edges in the image (second term). Additional features can easily be incorporated.

\section{Elastic Model Incorporating Statistical Shape}

While elastic models are useful in non-rigid registration, they are limited by themselves because they are too generic. With statistical information, we have a stronger bias to augment the elastic model. Statistical models can be powerful tools to directly capture the character of the variability of the individuals being modeled. Instead of relying on an elastic model to guide the deformation in a roughly plausible way, the statistics of a sample of images can be used to guide the deformation in a way governed by the measured variation of individuals. Thus, this paper proposes an algorithm which uses an elastic model, yet incorporates a statistical shape model to constrain solutions to more anatomically consistent deformations.

We pose the above displacement estimation problem in a maximum a posteriori framework. As input to the problem, we have both the intensity image of the study (individual), $I_{s}(\boldsymbol{w})$, and the boundary points of the study $\boldsymbol{b}_{s}(\mathbf{p}, n)=\left(x_{s}(\mathbf{p}, n), y_{s}(\mathbf{p}, n)\right)$, for $n=1,2, \cdots, N$, given the shape and pose parameters, $\mathbf{p}$, which are derived from the statistical shape model based boundary finding [16]. Thus, we want to maximize:

$$
\operatorname{Pr}\left(\boldsymbol{u} \mid I_{s}, \boldsymbol{b}_{s}(\mathbf{p})\right)=\frac{\operatorname{Pr}\left(\boldsymbol{u}, I_{s}, \boldsymbol{b}_{s}(\mathbf{p})\right)}{\operatorname{Pr}\left(I_{s}, \boldsymbol{b}_{s}(\mathbf{p})\right)}
$$

Ignoring the denominator, which does not change with $\boldsymbol{u}$, and by using Bayes rule, our aim is to find:

$$
\begin{aligned}
\arg \max _{\boldsymbol{u}} \operatorname{Pr}\left(\boldsymbol{u} \mid I_{s}, \boldsymbol{b}_{\boldsymbol{s}}(\mathbf{p})\right) & \equiv \arg \max _{\boldsymbol{u}} \operatorname{Pr}\left(\boldsymbol{b}_{\boldsymbol{s}}(\mathbf{p}) \mid \boldsymbol{u}, I_{\boldsymbol{s}}\right) \operatorname{Pr}\left(I_{s} \mid \boldsymbol{u}\right) \operatorname{Pr}(\boldsymbol{u}) \\
& \propto \arg \max _{\boldsymbol{u}} \operatorname{Pr}\left(\boldsymbol{b}_{\boldsymbol{s}}(\mathbf{p}) \mid \boldsymbol{u}\right) \operatorname{Pr}\left(I_{\boldsymbol{s}} \mid \boldsymbol{u}\right) \operatorname{Pr}(\boldsymbol{u}) \\
& \equiv \arg \max _{\boldsymbol{u}}\left[\ln \operatorname{Pr}(\boldsymbol{u})+\ln \operatorname{Pr}\left(I_{s} \mid \boldsymbol{u}\right)+\ln \operatorname{Pr}\left(\boldsymbol{b}_{s}(\mathbf{p}) \mid \boldsymbol{u}\right)\right](6)
\end{aligned}
$$

where (5) is true if we ignore the dependence of $\boldsymbol{b}_{s}(\mathbf{p})$ on $I_{s}$ because $\boldsymbol{b}_{s}(\mathbf{p})$ is obtained as a prior here and is not modified in this formulation. In the last equation, we have just taken the natural logarithm, which is a monotonically increasing function.

It is straightforward to directly connect the Bayesian posterior to the PDE in Eq.(2) [5]. This view is based on a variational principle from which the PDE can be derived. Such principles are well known in mechanics [12] and link the PDE formulation as the minimizer of some potential. The PDE for the linear elastic model, which is given in Eq.(2), is produced by setting the variation of the generalized Lagrangian energy density associated with constraints imposed by the linearized mechanics equal to zero [5]. The forcing function in the PDE (Eq. (2)) is then the variation of the likelihood function with respect to the vector displacement field [1] [10].

The first term in Eq.(6) corresponds to the transformation prior term, which is defined to give a high probability to transformations consistent with the elastic 
model and low probability to all other transformations. As mentioned above, it is a PDE of the deformation field, $u$, and is given by Eq.(2).

The second term in Eq.(6) is actually the likelihood term which depends on the study image. Let $I_{a}(\boldsymbol{w})$ be the intensity image of the atlas. We model the study image as a Gaussian process with mean given by the deformed atlas image, $I_{a}(\boldsymbol{w}-u(w))[5]$ (since Eulerian reference frame is used here, a mass particle instantaneously located at $\boldsymbol{w}$ originated from point $\boldsymbol{w}-\boldsymbol{u}(\boldsymbol{w}))$. That is,

$$
\ln \operatorname{Pr}\left(I_{s} \mid \boldsymbol{u}\right)=-\frac{1}{2 \sigma_{1}^{2}} \int_{\Omega}\left[I_{s}(\boldsymbol{w})-I_{a}(\boldsymbol{w}-u(\boldsymbol{w}))\right]^{2} d \boldsymbol{w}
$$

where $\sigma_{1}$ is the standard deviation of the Gaussian process.

The first body force, $\boldsymbol{F}_{1}$, is the gradient of this likelihood term with respect to $u$ at each $w[5]$ and is given by:

$$
\boldsymbol{F}_{1}(\boldsymbol{u})=-\frac{1}{\sigma_{1}^{2}}\left[I_{s}(\boldsymbol{w})-I_{a}(\boldsymbol{w}-u(\boldsymbol{w}))\right] \nabla I_{a}(\boldsymbol{w}-u(\boldsymbol{w}))
$$

This force is a combination of $I_{s}(w)-I_{a}(w-u(w))$, the difference in intensity between the study and the deformed atlas, and $\nabla I_{a}(\boldsymbol{w}-u(\boldsymbol{w})$, the gradient of the deformed atlas. The gradient term determines the directions of the local deformation forces applied to the atlas. As explained in the introduction, this kind of forcing by itself is often under-constrained.

The main contribution of this paper lies in the last term of Eq.(6), which incorporates statistical shape information into the non-rigid registration framework. The extra constraint of corresponding boundary points is used as an additional matching criterion. The boundary point positions are the result of the deformation of the model to fit the data in ways consistent with the statistical shape models derived from the training set, as described in Section 3 . Let $\boldsymbol{b}_{a}(n)=\left(x_{a}(n), y_{a}(n)\right)$, for $n=1,2, \cdots, N$, denote the atlas boundary points positions, which are known since we have full information about the atlas. We now model $\boldsymbol{b}_{s}(\mathbf{p})$ as a Gaussian process with mean given by the deformed atlas boundary position, expressed as $b_{a}(n)+u(w)$, for pixels $w$ on the deformed atlas boundary points. Then,

$$
\ln \operatorname{Pr}\left(\boldsymbol{b}_{s}(\mathbf{p}) \mid \boldsymbol{u}\right)=-\frac{1}{2 \sigma_{2}^{2}} \sum_{n=1}^{N}\left\|\boldsymbol{b}_{s}(\mathbf{p}, n)-\left[\boldsymbol{b}_{a}(n)+u(w)\right]\right\|^{2}
$$

where $\sigma_{2}$ is again the standard deviation of the Gaussian process.

The second body force, $\boldsymbol{F}_{2}$, is then the gradient of Eq.(9) with respect to $u$ for pixels $\boldsymbol{w}$ on the deformed atlas boundary points:

$$
\boldsymbol{F}_{2}(\boldsymbol{u})=\frac{1}{\sigma_{2}^{2}}\left\|\boldsymbol{b}_{s}(\mathbf{p}, n)-\left[\boldsymbol{b}_{a}(n)+\boldsymbol{u}(\boldsymbol{w})\right]\right\|
$$

$\boldsymbol{F}_{2}(u)$ is zero for pixels $\boldsymbol{w}$ not on the deformed atlas boundary points.

From Eq.(10), we can see that the calculated displacements at the sparse boundary points are constrained to match the vector difference of the corresponding atlas and study boundary point positions. This kind of forcing contains information from the statistical shape model. The result will match shape 
(a)

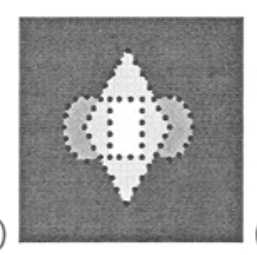

(b)

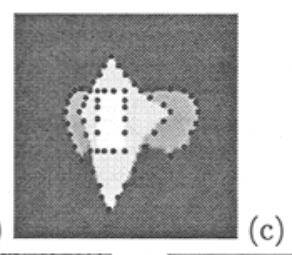

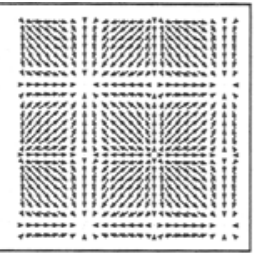

(d)

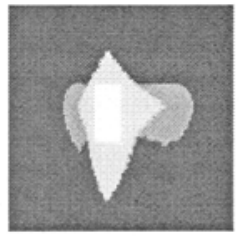

(e)

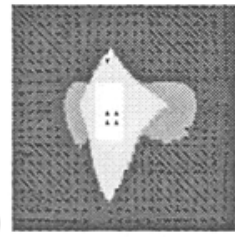

(f)

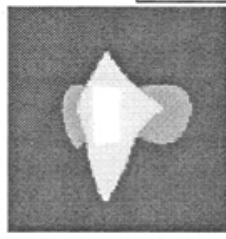

(g)

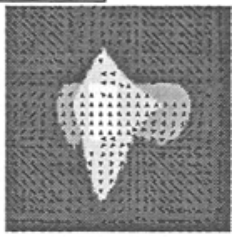

Fig. 3. Synthetic images $(100 \times 100)$ and displacement vectors. (a): atlas image with its control points; (b): study image with its control points; (c): true displacement vectors by Eq.(12); (d): our deformed atlas image; (e): errors in our estimated vectors on study image; (f): deformed atlas image by Christensen\&Miller's elastic method; (g): errors in estimated vectors for (f) on study image.
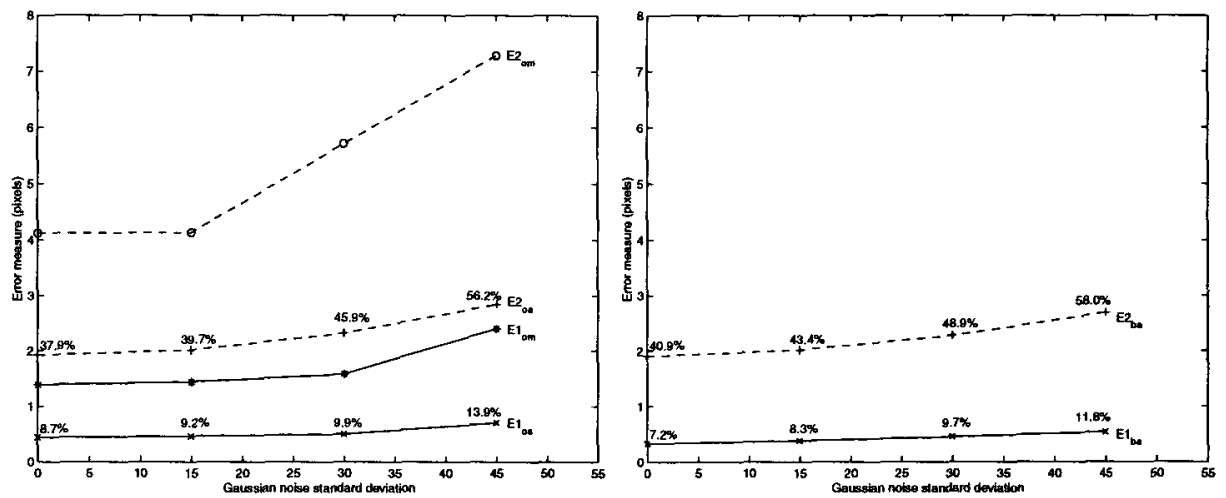

Fig. 4. Sensitivity to noise experiment for synthetic images. $E_{o m}$ : maximum displacement error over the deformed objects; $E_{o a}$ : average displacement error over the deformed objects; $E_{b a}$ : average displacement error on the sparse boundary points. Note: $E 1$ and $E 2$ are error measures by our method and Christensen\&Miller's elastic method respectively; the percentages shown are the percentages of the average errors $\left(E_{o a}, E_{b a}\right)$ relative to the true average displacements; the intensity range in the atlas and study images in Fig. 3 is 50 to 250.

features of the atlas and the study, such as high curvature points and important anatomical landmarks, in addition to the intensity measure.

The total force term, $\boldsymbol{F}(\boldsymbol{u})$, in Eq.(2) is then the weighted sum of $\boldsymbol{F}_{\mathbf{1}}(\boldsymbol{u})$ in Eq. (8) and $\boldsymbol{F}_{2}(\boldsymbol{u})$ in Eq. (10), that is, for each $\boldsymbol{w}$,

$$
\boldsymbol{F}(\boldsymbol{u})=c_{1} \boldsymbol{F}_{\mathbf{1}}(\boldsymbol{u})+c_{2} \boldsymbol{F}_{2}(\boldsymbol{u})
$$

The two coefficients, $c_{1}$ and $c_{2}$, can be related to the image contrast and the deformation between the atlas and study image. If $c_{2}$ is too large, $\boldsymbol{F}_{2}(\boldsymbol{u})$ will 
play a dominant role by matching only boundary points, which may cause discontinuity when the boundary points are not dense enough. On the other hand, if $c_{1}$ is too large, $\boldsymbol{F}_{2}(\boldsymbol{u})$ will have almost no effect and the algorithm is then an elastic regularization method (as [10]) without statistical information. For the time being, they are fixed empirically so that $\boldsymbol{F}_{1}(\boldsymbol{u})$ and $\boldsymbol{F}_{2}(\boldsymbol{u})$ are of the same order and then have the best effect.

(a)

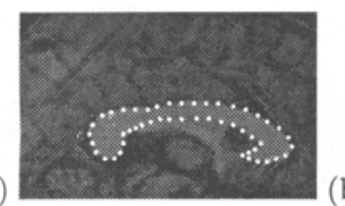

(b)
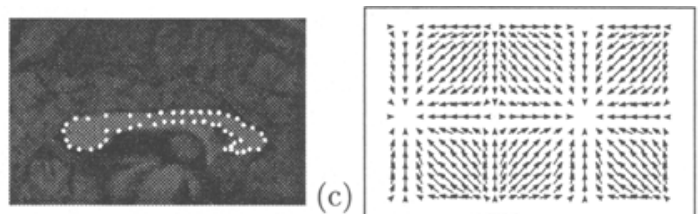

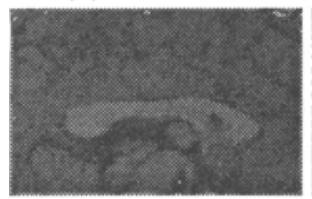

(d)

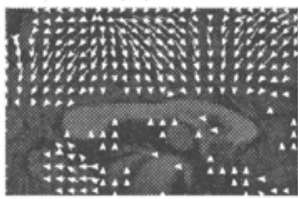

(e)

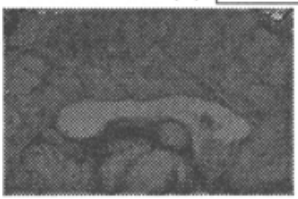

(f)

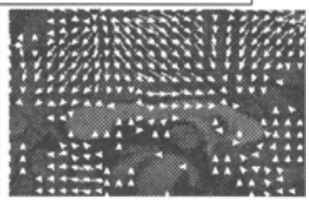

(g)

Fig. 5. MR sagittal corpus callosum images $(100 \times 64)$ and synthetic displacement vectors. (a): atlas image with control points; (b): study image with control points; (c): true displacement vectors; (d): our deformed atlas image; (e): errors in our estimated vectors on study image; (f): deformed atlas image by Christensen\&Miller's elastic method; $(\mathrm{g})$ : errors in estimated vectors for (f) on study image.

We have presented an elastic model based non-rigid registration procedure that incorporates information obtained from statistical shape model based boundary finding. Our non-rigid registration method is then composed of Eq.(2) and Eq.(11). In order to solve the problem, we discretize the two equations and solve the resulting system iteratively by using successive over-relaxation (SOR). The value of the total body force is used as the stopping criterion for the iterations.

\section{Experimental Results}

For all of the experiments, we apply Christensen\&Miller's elastic registration [10] [4] for a direct comparison based on our own implementation. We discretize and solve the resulting system iteratively by using SOR instead of stochastic gradient search, as in [10]. As to the computation time, while our method requires an extra force $\boldsymbol{F}_{2}$ calculation at sparse boundary points, this leads to faster and accurate convergence. Also, since the boundary finding step takes negligible time, i.e. only several seconds, the total convergence time of our method is usually a little faster.

\subsection{Evaluation Criterion}

To evaluate the methodology, we quantify errors in the displacement field over the objects of interest, since warping of the background is irrelevant. 
(b)

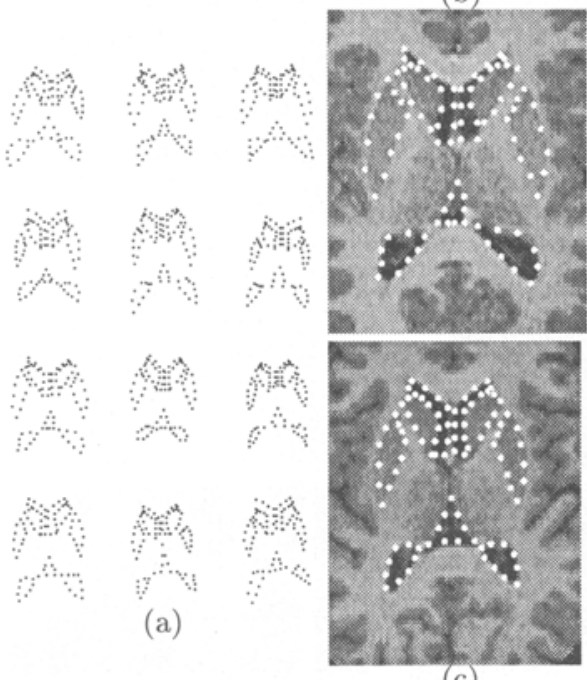

(c)
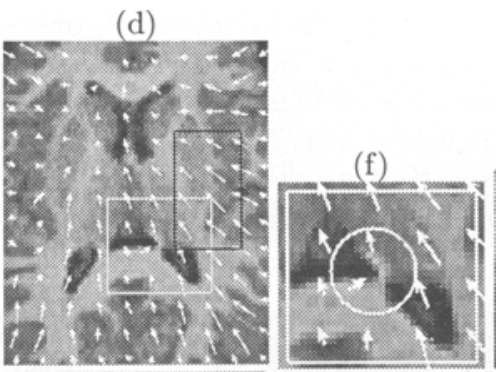

(h)

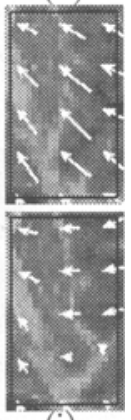

(i)

Fig. 6. MR axial brain images $(80 \times 100)$ and displacement vectors. (a): 12 examples of brain shapes from a training set with each example a 93 point model of basal ganglia and ventricle boundaries; (b): atlas image with its control points; (c): study image with its control points derived from our boundary finding algorithm [16]; (d): our estimated vectors over our deformed atlas; (e): estimated vectors by Christensen\&Miller's elastic method over their deformed atlas; (f): enlargement of (d) showing correct mapping of the ventricle corners; $(\mathrm{g})$ : mis-matching due to Christensen\&Miller's method; $(\mathrm{h})$ : well deformed putamen by our method (cropped); (i): poorly deformed putamen by Christensen\&Miller's method.

\begin{tabular}{|c|c|c|c|}
\hline Image (Fig.) & Error & Our method & Christensen\&Miller's method \\
\hline \multirow{3}{*}{$\begin{array}{c}\text { MR sagittal } \\
\text { corpus callosum } \\
\text { (Fig.5) }\end{array}$} & $\overline{E_{o a}(\%)}$ & 0.48 pixels $(11.5 \%)$ & 1.26 pixels $(30.0 \%)$ \\
\hline & $E_{\text {om }}$ & 1.33 pixels & 3.56 pixels \\
\hline & $E_{b a}(\%)$ & 0.45 pixels $(10.3 \%)$ & 1.51 pixels $(34.7 \%)$ \\
\hline$\overline{\mathrm{MR}}$ axial brain (Fig.6) & $\tilde{E}_{b a}(\%)$ & 0.75 pixels $(16.0 \%)$ & ixels $(43.7 \%)$ \\
\hline MR heart (Fig.7) & $E_{b 2}(\%)$ & 0.90 pixels $(15.4 \%)$ & 1.81 pixels $(31.1 \%)$ \\
\hline
\end{tabular}

Table 1. Error measure for MR corpus callosum images with known warping, real axial brain and heart images. $E_{o a}$ : average displacement error over corpus callosum; $E_{o m}$ : maximum displacement error over corpus callosum; $E_{b a}$ : average displacement error on sparse boundary points. Note: the percentages shown with each average error are with respect to the true average displacement.

Given a known warp, we can measure detailed displacement errors throughout the object. For testing purposes, we can define a particular warp and apply it to an image generating a warped study image to which the algorithm can be applied. We use the following sinusoidal displacement field for transforming the atlas image to a study image (see Fig.3(c) or Fig.5(c)):

$$
x_{n e w}=x_{o l d}+A_{x} \sin \left(\pi x_{o l d} / 32\right) ; \quad y_{n e w}=y_{o l d}+A_{y} \sin \left(\pi y_{o l d} / 32\right)
$$




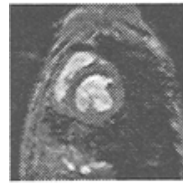

(a)

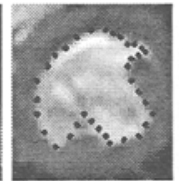

(b)

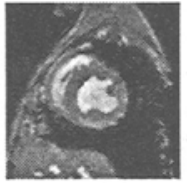

(c)

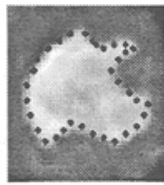

(d)

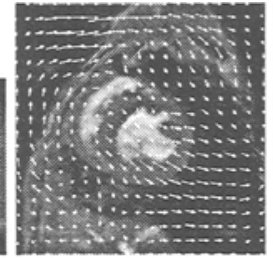

(e)

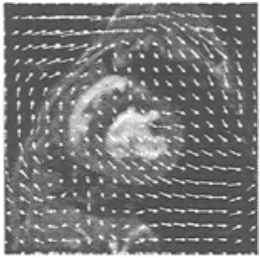

(f)

Fig. 7. MR heart images $(150 \times 150)$ and displacement vectors. (a): atlas image; (b): atlas image with its control points on the endocardium (cropped); (c): study image; (d): study image with control points derived from our boundary finding algorithm; (e): our estimated vectors over our deformed atlas image; (f): estimated vectors by Christensen\&Miller's elastic method over their deformed atlas image.

where $x_{\text {old }}$ and $y_{\text {old }}$ are coordinates of a point in the atlas image and $x_{n e w}$ and $y_{n e w}$ are coordinates of the corresponding point in the transformed study image. $A_{x}$ and $A_{y}$ are the limits of the maximum displacement distances along the $x$ and $y$ directions.

For a known non-rigid warp, the average $\left(E_{o a}\right)$ and maximum $\left(E_{o m}\right)$ differences between the estimated and actual displacement vectors over the objects are used to measure accuracy. We also use the average difference between the estimated and actual displacement vectors on the sparse boundary control points, $E_{b a}$. Since the control points are also derived from the known warp, all three measures only reflect the non-rigid registration, and do not include the boundary finding step. For true image pairs, we only use $E_{b a}$ as an accuracy measure because we do not know the true warp, except at sparse boundary points determined by an expert. Since the study image boundary control points are derived by our statistical shape model based boundary finding, the error, $E_{b a}$, for true image pairs includes both the boundary finding step and the non-rigid registration step.

\subsection{Synthetic Images with Known Warping}

The synthetic study image, Figure 3(b), is obtained by resampling the synthetic atlas image, Fig.3(a), based on the predetermined displacement vectors (Eq.(12)). The study control points are also derived from the same predetermined displacement vectors. The atlas image is then registered to the synthetic study image using our image registration procedure. Although the resulting deformed atlases for our method and Christensen\&Miller's method are similar, the estimated displacement vectors are not. From the errors in the estimated vectors (differences between the estimated and true displacement vectors), we can see that our method has almost zero error over the objects, while there is significant error in their approach.

The following experiment, shown in Fig.4, demonstrates the effect of noise on the two methods, by adding varying amounts of zero mean Gaussian noise to the synthetic images shown in Fig.3(a) and Fig.3(b). The error measures defined above are computed for our method $\left(E 1_{o a}, E 1_{o m}, E 1_{b a}\right)$ and Christensen\&Miller's elastic approach $\left(E 2_{o a}, E 2_{o m}, E 2_{b a}\right)$. 


\subsection{Real Image with Known Warping}

In this experiment (Fig.5), we apply a known warping (Eq.(12)) to a magnetic resonance (MR) sagittal brain image showing the corpus callosum. While the deformed atlases appear similar, the results (Fig.5 and Table 1) show that our method leads to a much better registration in the object of interest than Christensen\&Miller's elastic method.

\subsection{Real Atlas and Study Images}

Results of the method applied to MR brain (axial) and heart image pairs are shown in Fig. 6 and Fig.7. These are 2D slices roughly corresponding from different brains and hearts for demonstration purposes. The control points of the study image are derived from statistical shape model based boundary finding algorithm [16]. The shape model used for the brain examples incorporates multiple objects and thus also models the distance between the objects. From the error measures shown in Table 1, we see that even with the error in the boundary finding step, the final error of our method is still much better than Christensen\&Miller's elastic method. Note particular in Fig.6, the corner of the third ventricle in the study was not registered to the atlas correctly by Christensen\&Miller's method (Fig.6(g)). The structures of the study are shifted away from the corresponding ones in the atlas based on gray level information. Our method calculated the correct mapping (Fig.6(f)) by incorporating statistical shape information and using the corresponding boundary points as an extra constraint. Also note that the putamen did not deform well in Christensen\&Miller's method (Fig.6(i)) because the contrast of the putamen is too low compared to the contrast of the ventricles. In our approach, the putamen deformed correctly (Fig.6(h)) since shape information of the putamen was included.

\section{Conclusions}

This work presents a systematic approach for non-rigid registration. Transformations are constrained to be consistent with physical deformations of elastic solids in order to maintain the topology, or integrity, of the anatomic structures while having high spatial dimension to accommodate complex anatomical details. Both intensity information and statistical shape information are used as matching criteria in a Bayesian formulation. The incorporation of statistical shape information into the framework is the main contribution of our work. From the experimental results, statistical boundary shape information has been shown to augment and improve an elastic model formulation for non-rigid registration.

Our current and future directions include exploring the use of fluid models to track long-distance, nonlinear deformations [17] and generalization to 3D. Although finding the landmark surface points and model construction in 3D will require new strategies, generalizing the purely elastic model deformation to 3D is straightforward and has been developed by Christensen et al. [4]. Of course, the computational cost increases with the number of voxels. 


\section{References}

1. Y. Amit, U. Grenander and M. Piccioni, "Structural image restoration through deformable templates," J. American Statistical Association, Vol. 86, no. 414, pp. 376-387, June 1991.

2. R. Bajcsy and S. Kovacic, "Multiresolution elastic matching," Computer Vision, Graphics and Image Processing, Vol. 46, pp. 1-21, 1989.

3. F. L. Bookstein, "Shape and information in medical images: A decade of the morphometric synthesis," Proc. Workshop Math. Meth. Biomed. Image Anal., pp. 2-12, June 1996.

4. G. E. Christensen, M. I. Miller and M. W. Vannier, "Individualizing neuroanatomical atlases using a massively parallel computer," Computer, pp. 32-38, January 1996.

5. G. E. Christensen, R. D. Rabbitt and M. I. Miller, "Deformable templates using large deformation kinematics," IEEE Trans. on Image Processing, Vol. 5, no. 10, pp. 1435-1447, October 1996.

6. D. L. Collins, A. C. Evans, C. Holmes and T. M. Peters, "Automatic 3D segmentation of neuro-anatomical structures from MRI," Information Processing in Medical Imaging, pp. 139-152, 1995.

7. T. F. Cootes, C. J. Taylor, D. H. Cooper and J. Graham, "Active shape models their training and application," Computer Vision and Image Understanding, Vol. 61, no. 1 , pp. 38-59, 1995.

8. C. Davatzikos and J. Prince, "Brain image registration based on curve mapping," IEEE Workshop Biomedical Image Anal., pp. 245-254, 1994.

9. J. Gee, L. L. Briquer, C. Barillot and D. Haynor, "Probabilistic matching of brain images," Information Processing in Medical Imaging, pp. 113-125, 1995.

10. M. I. Miller, G. E. Christensen, Y. Amit and U. Grenander, "Mathematical textbook of deformable neuroanatomies," Proc. National Academy of Science, Vol. 90, no. 24, pp. 11944-11948, 1993.

11. R. D. Rabbitt, G. E. Christensen and M. I. Miller, "Mapping of hyperelastic deformable templates using finite element method," Vision Geometry IV, Vol. 2573, pp. 252-265, July 1995.

12. J. N. Reddy, Energy and Variational Methods in Applied Mechanics, New York: Wiley-Interscience, 1984.

13. L. H. Staib and J. S. Duncan, "Boundary finding with parametrically deformable models," IEEE Trans. Pattern Analysis and Machine Intelligence, Vol. 14, no. 11, pp. 1061-1075, 1992.

14. G. Székely, A. Keleman, C. Brechbuler and G. Gerig, "Segmentation of 2-D and 3$\mathrm{D}$ objects from MRI volume data using constrained elastic deformations of flexible Fourier contours and surface models," Medical Image Analysis, Vol. 1, no. 1, pp. 19-34, 1996.

15. J. P. Thirion, "Non-rigid matching using demons," Proc. Conf. Computer Vision and Pattern Recognition, pp. 245-251, June 1996.

16. Y. Wang and L. H. Staib, "Boundary finding with correspondence using statistical shape models," Proc. Conf. Computer Vision and Pattern Recognition, pp. 338-345, Santa Barbara, California, June 1998.

17. Y. Wang and L. H. Staib, "Integrated approaches to non-rigid registration in medical images," Fourth IEEE Workshop on Applications of Computer Vision, in press, Princeton, New Jersey, October 1998. 